\title{
Ecological Psychology: Diagnosing and Treating Patients in Complex Environments
}

\section{Michelle Daniel, Dario Torre, Steven J Durning, Eric Wilson, Joseph Rencic}

According to the theory of ecological psychology, providers diagnose and treat patients through interactions with complex environments. Each environment offers providers unique affordances (i.e., opportunities to make an accurate and timely diagnosis without error). Each provider also brings unique effectivities (i.e., knowledge, skills and abilities to take action on those opportunities). Interactions with the environment are shaped by participants' intentions (i.e., their goals to diagnose and treat patients), as well as attentions (i.e., their recognition of affordances of the case or of the environment).

Consider the following: A 45-year-old male presents to the emergency department with fever and altered mental status. He is breathing rapidly and vomiting intermittently. He is unable to provide a history. The resident pulls up the patient's chart and notices a history of insulin-dependent diabetes mellitus (IDDM) and IV drug abuse (IVDA). The nurse checks a glucose and notes it is $>600$. The resident physician smells ketones on the patient's breath and observes Kussmaul breathing. She makes a diagnosis of diabetic ketoacidosis (DKA). She orders an ECG, but fails to notice $U$ waves indicative of hypokalemia as she was looking for signs of myocardial infarction (MI). She orders insulin and fluids, an error that may precipitate torsades. Due to the noisy environment, she doesn't detect the tricuspid murmur indicative of possible right-sided endocarditis as the precipitant of DKA.

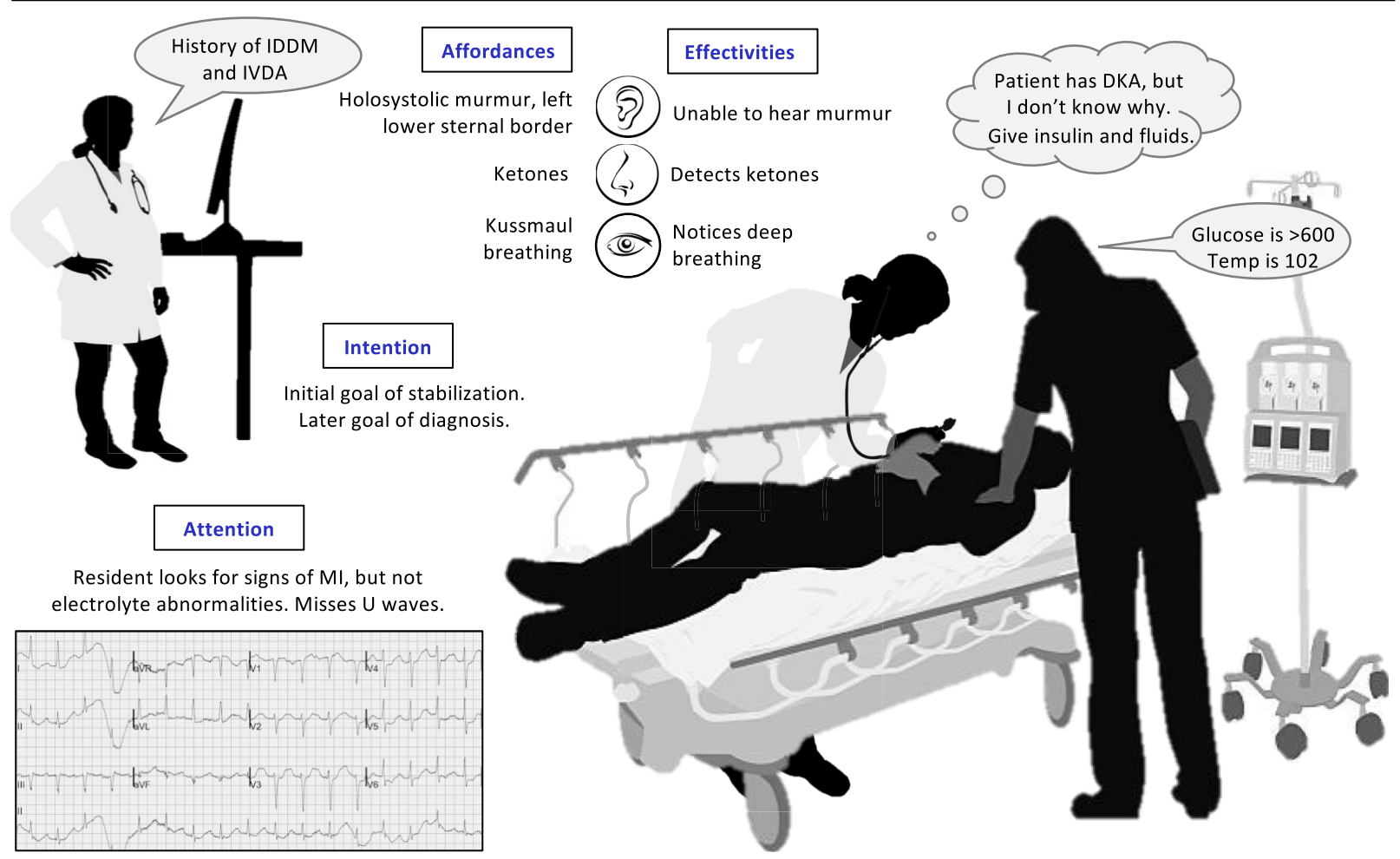

This case highlights the interplay between affordances, effectivities, intention and attention on reasoning and error. Multiple affordances in the environment are either acted upon or missed based on the resident's effectivities (e.g., smells ketones, sees Kussmaul respirations and diagnoses DKA, but cannot appreciate the murmur). The resident's immediate intention is to stabilize the patient. Her focus is on bringing the glucose down, causing her to rush to give insulin before considering the patient's potassium level. This highlights why attention is critical to identify all the information afforded by the EKG. When a patient presents with chest pain, attention should focus on signs of ischemia. When a patient presents with DKA, one must look for signs of hypo- or hyperkalemia, as starting insulin too early can precipitate torsades. After the resident carefully considers the precipitant of this patient's DKA, she might consider endocarditis. At that point, if she returns to listen to the patient's heart sounds with properly "tuned" attention and the affordance of a quiet room, she might appreciate the murmur and diagnosis endocarditis (effectivities).

References: 1) Gibson, EJ. (2000). Perceptual Learning and Development: An Ecological Approach. New York, New York: Oxford university Press.

Disclaimer: The views expressed herein are those of the authors and not necessity those of the Department of Defense or other federal agencies. 
*Corresponding author: Michelle Daniel, Office of Medical Student Education, University of Michigan Medical School, 6123 Taubman Health Sciences Library, 1135 Catherine, Ann Arbor, 48109-0624, MI, USA, E-mail: micdan@umich.edu

Dario Torre and Steven J. Durning: Internal Medicine, Uniformed Services University of the Health Sciences, Bethesda, MD, USA Eric Wilson: Medical Student, University of Michigan Medical School, Ann Arbor, MI, USA
Joseph J. Rencic: Internal Medicine, Boston University School of Medicine, Boston, MA, USA. https://orcid.org/0000-0002-2598-3299

https://doi.org/10.1515/dx-2020-0008

Published online June 25, 2020

Supplementary Material: The licenses for the images are provided in the online version of this article (https://doi.org/10.1515/dx-2020-0008). 